\title{
Furosemide response predicts acute kidney injury in children after cardiac surgery
}

Jamie Penk, MD, ${ }^{\mathrm{a}}$ Katja M. Gist, DO, MSc, ${ }^{\mathrm{b}}$ Eric L. Wald, MD, MSCI, ${ }^{\mathrm{c}}$ Laura Kitzmiller, MD, ${ }^{\mathrm{d}}$

Tennille N. Webb, MD, ${ }^{\mathrm{e}} \mathrm{Yi}$ Li, MS, ${ }^{\mathrm{a}}$ David S. Cooper, MD, MPH, ${ }^{\mathrm{f}}$ Stuart L. Goldstein, MD, ${ }^{\mathrm{f}}$ and Rajit K. Basu, MD, MS

\section{ABSTRACT}

Objective: A standardized assessment of response to furosemide is predictive of acute kidney injury progression in adults, but a paucity of data exists in pediatric patients. We evaluate furosemide responsiveness in a multicenter cohort of pediatric patients after cardiac surgery.

Methods: Children who underwent cardiac surgery with a Society of Thoracic Surgeons-European Association for Cardiothoracic Surgery score of 3 or greater were retrospectively identified. The first dose of furosemide after surgery was recorded, and hourly urine output for 6 hours was recorded after the index dose. Urine flow rate calculated as urine output per hour was used to predict development of acute kidney injury.

Results: A total of 166 patients from 4 institutions (median age, 6.3 months; interquartile range, 0.4-27.7) were included. Acute kidney injury occurred in 54 patients $(33 \%)$. Compared with those without acute kidney injury, the 2 - and 6-hour urine flow rates were significantly lower in patients in whom acute kidney injury developed: $2.9(0.9-6.5)$ versus $5.0(2.5-9.0) \mathrm{mL} / \mathrm{kg} / \mathrm{h}$ for 2 -hour urine flow rate, $P=.004$, and 2.4 (1.2-4.0) versus $4.0(2.3-5.9) \mathrm{mL} / \mathrm{kg} / \mathrm{h}$ for 6-hour flow rate, $P=.001$. In multivariable regression analysis, 2 -hour (odds ratio, $1.2, P=.002$ ) and 6-hour (odds ratio, 1.40, $P<.001$ ) urine flow rates were independently associated with acute kidney injury development. Lower urine flow rate at both 2 and 6 hours was also independently associated with longer hospital length of stay.

Conclusions: Lower urine flow rate after furosemide administration, when evaluated in a heterogeneous cohort of children from multiple institutions after pediatric cardiac surgery, was independently associated with subsequent acute kidney injury and longer length of stay. Future prospective studies are needed to validate furosemide responsiveness as a predictor of acute kidney injury. (J Thorac Cardiovasc Surg 2019;157:2444-51)

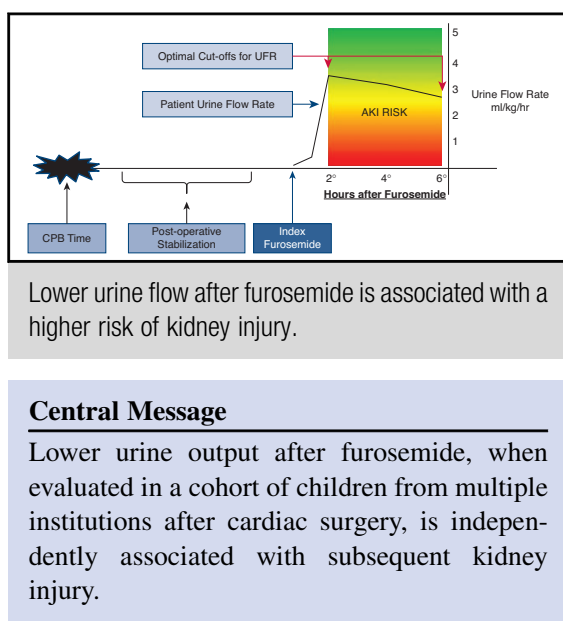

Perspective

AKI occurs frequently after pediatric cardiac surgery. Prediction of kidney injury may expedite preventive therapy. This study demonstrates that across multiple treatment strategies and a wide range of ages, low urine output after furosemide is associated with subsequent kidney injury. Development of a pediatric FST could provide an early predictor of kidney injury.

See Commentary on page 2452 .
Acute kidney injury (AKI) occurs frequently in critically ill neonates and children. Recent large epidemiologic data identify an AKI incidence of $26 \%$ to $30 \%$ within the first 7 days in children admitted to neonatal and pediatric intensive care units, and the severe AKI (stage $\geq 2$ ) rate identified is $11.6 \%$ to $16.0 \% .^{1-3}$ This incidence is important as

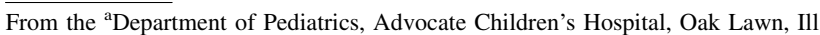
${ }^{\mathrm{b}}$ University of Colorado Denver, Anschutz Medical Campus, Department of Pediatrics, Division of Pediatric Cardiology, Children's Hospital Colorado, Aurora, Colo; ${ }^{\mathrm{c}}$ Northwestern University, Department of Pediatrics, Division of Pediatric Critical Care, Ann and Robert H. Lurie Children's Hospital, Chicago, Ill; ${ }^{\mathrm{d}}$ Children's Hospital of Michigan, Detroit, Mich; 'University of Alabama at Birmingham, Department of Pediatrics, Division of Pediatric Nephrology, Children's of Alabama, Birmingham, Ala; ${ }^{\mathrm{f}}$ University of Cincinnati, Department of Pediatrics, Center for Acute Care Nephrology, Cincinnati Children's Hospital and Medical Center, Cincinnati, Ohio; and ${ }^{\mathrm{g}}$ Emory University, Department of Pediatrics, Division of Critical Care Medicine, Children's Healthcare of Atlanta, Atlanta, Ga. intensive care unit morbidity increases in children with AKI. ${ }^{1-3}$ AKI may be even more common in children who undergo cardiac surgery because reported AKI ranges from $27 \%$ to $52 \% .^{3-5}$ In these patients, AKI is also associated with increased hospital morbidity, including increased duration of mechanical ventilation and intensive

\footnotetext{
Institutional Review Board number 6268 Institutional Review Board approval: February 22, 2015.

J.P. and K.M.G. contributed equally to the article.

Received for publication June 7, 2018; revisions received Nov 28, 2018; accepted for publication Dec 19, 2018; available ahead of print Feb 10, 2019.

Address for reprints: Jamie Penk, MD, 4440 95th St, Oak Lawn, IL 60453 (E-mail: jamiepenk@gmail.com). $0022-5223 / \$ 36.00$

Copyright (C) 2019 by The American Association for Thoracic Surgery https://doi.org/10.1016/j.jtcvs.2018.12.076
} 

Abbreviations and Acronyms
AKI = acute kidney injury
AUC $=$ area under the curve
$\mathrm{CPB}=$ cardiopulmonary bypass
FST $=$ furosemide stress test
$\mathrm{IQR}=$ interquartile range
ROC $=$ receiver operating characteristic
$\mathrm{SCr}=$ serum creatinine
STAT $=$ Society of Thoracic Surgeons-European
Association for Cardio-Thoracic Surgery
$\mathrm{UFR}=$ urine flow rate

Scanning this QR code will take you to the article title page to access supplementary information.

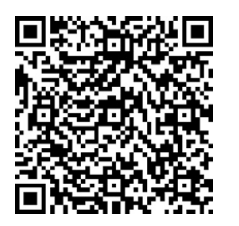

care and hospital lengths of stay, and higher rates of mortality.

Management options for AKI are limited. Mainstays of treatment are mostly supportive and include optimizing patient hemodynamics, reducing or eliminating nephrotoxic agents, being vigilant with regard to fluid balance, and administering renal replacement therapy. These steps are generally incorporated into patient care after AKI has already occurred. Delayed detection of AKI and an inability to reliably recognize incipient injury or predict severe injury may be responsible, in part, for poor AKI-related outcomes. Reliance on serum creatinine $(\mathrm{SCr})$ has welldocumented limitations. In addition to timing and steady-state measurement, the effects of hydration and muscle mass on creatinine may be of particular importance in children after cardiac surgery, in whom risk factors for fluid accumulation may be high (Video 1).

Alternative strategies to identify AKI before an increase in SCr may lead to improved patient outcomes. Approximately 2 decades of novel biomarker discovery and validation work demonstrate early detection and AKI prediction are possible, particularly in children after cardiac surgery. ${ }^{6}$ However, despite robust performance in these populations, most biomarkers are not routinely used in practice and few are clinically available or approved for use. This is unfortunate given that recent evidence suggesting simple renal protective measures triggered by biomarker-based detection are associated with reduced severe AKI rates and less severe patient morbidity. ${ }^{7}$ Functional tests of the kidney may be even more indicative of kidney health than injury biomarkers.

\section{The Continuum of AKI}

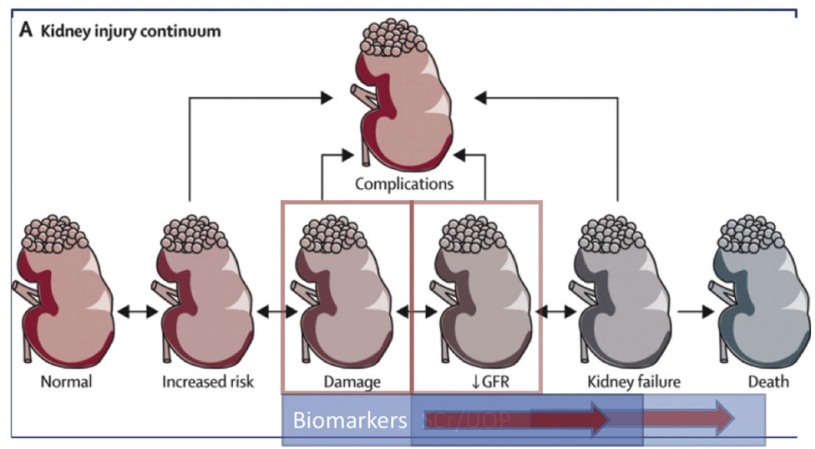

VIDEO 1. Dr Basu explains the risk of AKI after pediatric cardiac surgery and the benefits of early detection. Results of this study are discussed, and their relevance is explained. Video available at: https://www.jtcvs.org/ article/S0022-5223(19)30003-0/fulltext.

The furosemide stress test (FST) is a standardized assessment of urine output in response to furosemide and may reflect renal function. The FST tested in critically ill adults, defined by urine flow rate (UFR) 2 and 6 hours after furosemide administration, demonstrates high-level discrimination for AKI progression and ultimate need of renal replacement therapy. The predictive performance of the FST is superior to serum or urine AKI biomarkers. ${ }^{8}$ Limited data are published describing the FST in children even though the use of parenteral diuretics such as furosemide after pediatric cardiac surgery is ubiquitous.

We performed a multicenter study to test the hypothesis that a version of the FST evaluated retrospectively and without correction for fluid status was predictive of AKI in a heterogeneous group of children after cardiac surgery.

\section{MATERIALS AND METHODS \\ Population}

We performed a multicenter, retrospective chart review of consecutive children undergoing cardiac surgery requiring cardiopulmonary bypass (CPB) from January 2014 to July 2015. Four urban children's hospitals with pediatric cardiac surgery programs were included: Cincinnati Children's Hospital Medical Center, The Ann and Robert H. Lurie Children's Hospital of Chicago, Children's Hospital Colorado, and Advocate Children's Hospital. Inclusion criteria included all children from birth to 18 years with a Society of Thoracic Surgeons-European Association for Cardio-Thoracic Surgery (STAT) mortality score ${ }^{9}$ of 3 or greater. Exclusion criteria included gestational age less than 34 weeks, weight less than $2 \mathrm{~kg}$ at the time of surgery, administration of the first dose of furosemide in the operating room, those who did not receive furosemide or those with a delay in furosemide administration to after 7 days from surgery, use of a continuous furosemide infusion before or within 6 hours of a bolus dose, a preoperative glomerular filtration rate less than $25 \mathrm{~mL} / \mathrm{min} /$ $1.73 \mathrm{~m}^{2}$ before surgery, a history of renal transplant, or use of a peritoneal drain for dialysis before the first furosemide dose. Institutional review board approval was obtained at each site and included a waiver of informed consent. 


\section{Data Collection}

Demographic, preoperative, intraoperative, and postoperative data were collected from each patient electronic medical record. Baseline (preoperative) creatinine was defined as the lowest $\mathrm{SCr}$ within the 3 months before surgery. If none was available, one was calculated using the following previously validated equation, ${ }^{10}$ assuming an estimated glomerular filtration rate of $120 \mathrm{~mL} / \mathrm{min} / \mathrm{m}^{2}$ :

$$
\begin{gathered}
\text { Serumcreatinine }=(0.413 * \text { intensive care admission height }) \\
/ 120 \mathrm{~mL} / \mathrm{min} / 1.73 \mathrm{~m}^{2}
\end{gathered}
$$

Use of preoperative diuretics or nephrotoxic medications within 7 days of surgery was recorded. Nephrotoxic medications included nonsteroidal anti-inflammatory medications, specific antimicrobials, or angiotensinconverting enzyme inhibitors.

The adult FST was originally validated in adults with at least stage $1 \mathrm{AKI}$ by SCr by quantifying UFR for 2 hours after furosemide using a cutoff of 100 or $200 \mathrm{~mL} / \mathrm{h}$ after $1 \mathrm{mg} / \mathrm{kg}$ or $1.5 \mathrm{mg} / \mathrm{kg}$ (for furosemide-naïve patients or patients who previously received furosemide, respectively). ${ }^{11}$ After the FST, intravenous fluid was readministered in the quantity of volume urinated after furosemide so that patients maintained a net even fluid balance after the FST. Because our study was retrospective, all patients, regardless of AKI status, were assessed for eligibility on the basis of inclusion or exclusion criteria. The dose of furosemide ("index dose") was recorded in milligrams per kilogram. UFR was quantified hourly for 6 hours after index furosemide and reported as both total volume and weight adjusted flow $(\mathrm{mL} / \mathrm{kg} / \mathrm{h})$. The primary outcome was development of AKI defined using the Kidney Disease: Improving Global Outcomes $\mathrm{SCr}$ criteria ${ }^{12}$ during the first 7 days after the index furosemide dose. Severe AKI was denoted as at least stage 2 by change in $\mathrm{SCr}$ (a 100\% increase in SCr over baseline).

Patient outcome data collected included use of renal replacement therapy and extracorporeal membrane oxygenation, duration of mechanical ventilation and length of stay, and mortality.

\section{Statistical Analysis}

The primary outcome of interest was the development of AKI. Continuous variables were summarized using mean \pm standard deviation or median with interquartile range (IQR) for normally and non-normally distributed variables, respectively, and were compared between groups using $t$ tests or Mann-Whitney $U$ tests as appropriate. Categoric variables are presented as frequency with percent and compared using chi-square tests or Fisher exact tests. Multivariate logistic regression was used to examine the association between postoperative UFR and AKI. A set of predetermined candidate risk factors (including age, gender, weight for age $z$ score, STAT score, nephrotoxin exposure, intraoperative steroid use, lactate at admission, CPB duration, and furosemide dose) were concurrently adjusted for with UFR, and the final predictive model preserves only those that influenced the UFR-AKI relationship. A multiple Poisson regression was performed to determine how UFR influenced length of stay after adjusting for AKI, duration of ventilation, gender, age, weight, and STAT category. Finally, a receiver operating characteristic (ROC) curves analysis was performed to evaluate the performance of UFR as a predictor of the risk of developing severe AKI during the first 7 postoperative days. All statistical analyses were performed using SPSS 25 (SPSS Inc, Chicago, Ill) and SAS 9.4 (SAS Institute Inc, Cary, NC).

\section{RESULTS}

A total of 172 patients were eligible, of whom 6 patients were excluded because only a single measure of postoperative SCr was available. A total of 166 patients were included in the final analysis with a median age of 6.3 months (IQR, 0.4-27.7) (Table 1). There was a measured baseline creatinine for 145 patients and a baseline SCr imputed as described earlier for 23 patients.

\begin{tabular}{|c|c|c|c|}
\hline & No AKI $(n=112)$ & $\mathbf{A K I}(\mathbf{n}=\mathbf{5 4})$ & $P$ value \\
\hline \multicolumn{4}{|l|}{ Demographics } \\
\hline Age (mo), median (IQR) & $9.1(3.8-45.9)$ & $4.0(0.1-8.9)$ & $<.001$ \\
\hline Weight for age $z$ score, mean (SD) & $-1.2 \pm 1.6$ & $-0.6 \pm 3.6$ & .12 \\
\hline Gender (female), n (\%) & $58(51.8 \%)$ & $32(59.3 \%)$ & .37 \\
\hline Baseline creatinine (mg/dL), mean (SD) & $0.40 \pm 0.15$ & $0.29 \pm 0.18$ & $<.001$ \\
\hline Preoperative diuretic exposure, $\mathrm{n}(\%)$ & $18(16.1 \%)$ & $6(11.1 \%)$ & .40 \\
\hline Preoperative nephrotoxic medication, $\mathrm{n}(\%)$ & $40(35.7 \%)$ & $17(31.5 \%)$ & .59 \\
\hline \multicolumn{4}{|l|}{ Operative characteristics } \\
\hline Steroids used in surgery, $\mathrm{n}(\%)$ & $93(83.8 \%)$ & $43(82.7 \%)$ & .86 \\
\hline \multicolumn{4}{|l|}{ STAT score, $\mathrm{n}(\%)$} \\
\hline $\mathrm{V}$ & $4(4 \%)$ & $3(6 \%)$ & \\
\hline IV & $47(43 \%)$ & $29(54 \%)$ & .26 \\
\hline III & $58(53 \%)$ & $21(40 \%)$ & \\
\hline Crossclamp time (min), mean (SD) & $83.8 \pm 44.4$ & $79.1 \pm 56.9$ & .59 \\
\hline $\mathrm{CPB}$ duration (min), mean (SD) & $144.9 \pm 54.1$ & $142.0 \pm 64.5$ & .77 \\
\hline Admission lactate $(\mathrm{mmol} / \mathrm{L})($ missing data $\mathrm{n}=8)$, mean $(\mathrm{SD})$ & $2.8 \pm 1.8$ & $3.0 \pm 2.6$ & .42 \\
\hline \multicolumn{4}{|l|}{ Outcomes } \\
\hline Duration of ventilation (d), median (IQR) & $1(0-1)$ & $1(0-3)$ & .009 \\
\hline Length of stay (d), median (IQR) & $4(3-7)$ & $7(3.5-12.5)$ & .002 \\
\hline Mortality, n (\%) & $2(1.8 \%)$ & $1(1.9 \%)$ & .69 \\
\hline
\end{tabular}

TABLE 1. Demographics, operative, and postoperative characteristics of patients with and without acute kidney injury

$A K I$, Acute kidney injury; IQR, interquartile range; $S D$, standard deviation; STAT, Society of Thoracic Surgeons-European Association for Cardio-Thoracic Surgery; $C P B$, cardiopulmonary bypass. 


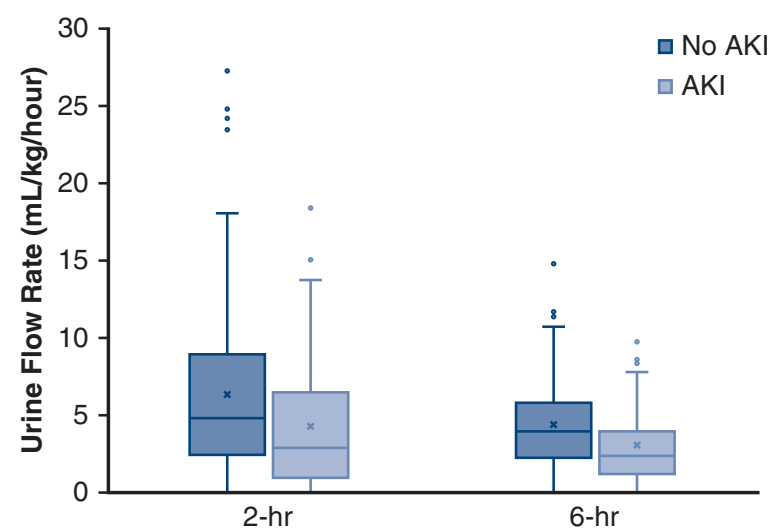

FIGURE 1. Comparison of median UFR through 2 and 6 hours between patients with and without AKI. The middle horizontal line represents the median flow rate, and the upper and lower borders of the box represent upper and lower quartiles. The upper and lower whiskers represent maximum and minimum values on nonoutliers, and the extra dots represent outliers. The median UFR was significantly lower in patients with AKI at both 2 and 6 hours after administration of the index furosemide dose. AKI, Acute kidney injury.

\section{Development of Acute Kidney Injury Was Common in Our Cohort}

Fifty-four patients (33\%) developed AKI (stage 1: 17\%, stage 2: $8 \%$ AKI, and stage 3: $8 \%$ ). No patients required renal replacement therapy. Patients with AKI were younger than patients without AKI (4 months [IQR, 0.1-8.9] vs 9.1 months [IQR, 3.8-45.9], $P<.001)$ and demonstrated a concomitant lower baseline $\mathrm{SCr}(P<.001)$. Patients with AKI had a longer duration of ventilation and increased length of stay compared with patients without AKI $(P=.009$ and $P=.002$, respectively) (Table 1).

\section{Urine Flow Rate After Furosemide Is Lower in Patients Who Developed Acute Kidney Injury}

The median time to furosemide administration from admission to the intensive care unit was 14.0 hours (IQR,

TABLE 2. The 2- and 6-hour urine flow rates in patients with and without acute kidney injury

\begin{tabular}{lccc}
\hline & $\begin{array}{c}\text { No AKI } \\
(\mathbf{n}=\mathbf{1 1 2})\end{array}$ & $\begin{array}{c}\text { AKI } \\
(\mathbf{n}=\mathbf{5 4})\end{array}$ & $\boldsymbol{P}$ value \\
\hline $\begin{array}{l}\text { Furosemide dose }(\mathrm{mg} / \mathrm{kg}), \\
\text { mean }(\mathrm{SD})\end{array}$ & $0.9 \pm 0.3$ & $0.9 \pm 0.3$ & .32 \\
$\begin{array}{l}\text { Peak creatinine }(\mathrm{mg} / \mathrm{dL}), \\
\text { mean }(\mathrm{SD})\end{array}$ & $0.44 \pm 0.16$ & $0.62 \pm 0.32$ & $<.001$ \\
$\begin{array}{l}\text { UFR (mL/kg/h) 0-2 h, median } \\
\quad(\mathrm{IQR})\end{array}$ & $5.0(2.5-9.0)$ & $2.9(0.9-6.5)$ & .004 \\
$\begin{array}{l}\text { UFR (mL/kg/h) 0-6 h, median } \\
(\mathrm{IQR})\end{array}$ & $4.0(2.3-5.9)$ & $2.4(1.2-4.0)$ & .001 \\
\hline
\end{tabular}

$\overline{A K I}$, Acute kidney injury; $S D$, standard deviation; $U F R$, urine flow rate; $I Q R$, interquartile range.
9.5-19.3). Two-hour UFR after furosemide administration was lower among patients who developed AKI $(2.9 \mathrm{~mL} /$ $\mathrm{kg} / \mathrm{h}$; IQR, 0.9-6.5) than among patients without AKI $(5.0 \mathrm{~mL} / \mathrm{kg} / \mathrm{h}$; IQR $2.5-9.0)(P=.004)$. Likewise, 6-hour UFR after furosemide administration was lower in patients who developed AKI (2.4 mL/kg/h; IQR, 1.2-4.0) compared with those who did not $(4.0 \mathrm{~mL} / \mathrm{kg} / \mathrm{h} ; \mathrm{IQR}, 2.3-5.9)$ $(P=.001)$ (Figure 1). There was no difference in the administered furosemide dose between patients and patients without AKI (Table 2). These associations were maintained when patients aged less than 1 month were removed from the analysis.

\section{Urine Flow Rate After Furosemide Is Independently Associated With Acute Kidney Injury}

Lower 2- and 6-hour UFR were independently associated with development of AKI after adjusting for sex, age, weight, steroid use before bypass, lactate, and STAT score (odds ratio, 1.2, $P=.002$ and odds ratio, 1.4, $P<.001$, respectively) (Figure 2). Every $1 \mathrm{~mL} / \mathrm{kg} / \mathrm{h}$ increase in the UFR during the first 6 hours reduced the odds of developing AKI by $41 \%(95 \%$ CI, 17-70).

\section{Lower Urine Flow Rate Is Associated With Worse Clinical Outcomes}

A multivariable regression analysis demonstrated that lower 2- and 6-hour UFR were associated with a longer length of stay after adjusting for AKI status, duration of ventilation, gender, STAT score, weight, and age $(P<.001, \beta=-0.09$ and $P<.001, \beta=-0.16$, respectively). Every $1 \mathrm{~mL} / \mathrm{kg} / \mathrm{h}$ decrease in 2- and 6-hour UFR increased the length of hospital stay by $9 \%$ and $15 \%$, respectively.

\section{Receiver Operating Characteristic Analysis of Urine Flow Rate for Prediction of Severe Acute Kidney Injury}

In an ROC analysis, severe AKI (AKI stage $\geq 2$ ) versus AKI 1 or less was used because it was thought to be more clinically relevant and would optimize the area under the curve (AUC). The AUC for severe AKI was $0.68(95 \%$ CI, 0.56-0.79) for 2-hour UFR and $0.69(95 \% \mathrm{CI}$, 0.58-0.81) for 6-hour UFR. A post hoc analysis of 111 patients with the first furosemide dose limited to 0.8 to $1.2 \mathrm{mg} / \mathrm{kg}$ did not improve the AUC for predicting severe AKI. Based on the ROC analysis, the optimal cut-point for predicting severe AKI was a 2-hour UFR $3.6 \mathrm{~mL} / \mathrm{kg} / \mathrm{h}$ or less (sensitivity $73 \%$, specificity $64 \%$ ). The optimal 6-hour UFR cut-point was $2.7 \mathrm{~mL} / \mathrm{kg} / \mathrm{h}$ or less (sensitivity $73 \%$, specificity $68 \%$ ). Of the patients who developed severe AKI, 17 of $26(65 \%)$ had lower urine output than these optimal cutoffs through both 2 and 6 hours. The positive predictive values for 2-hour UFR and 6-hour UFR are $27.1 \%$ and $30.2 \%$, respectively. The negative 

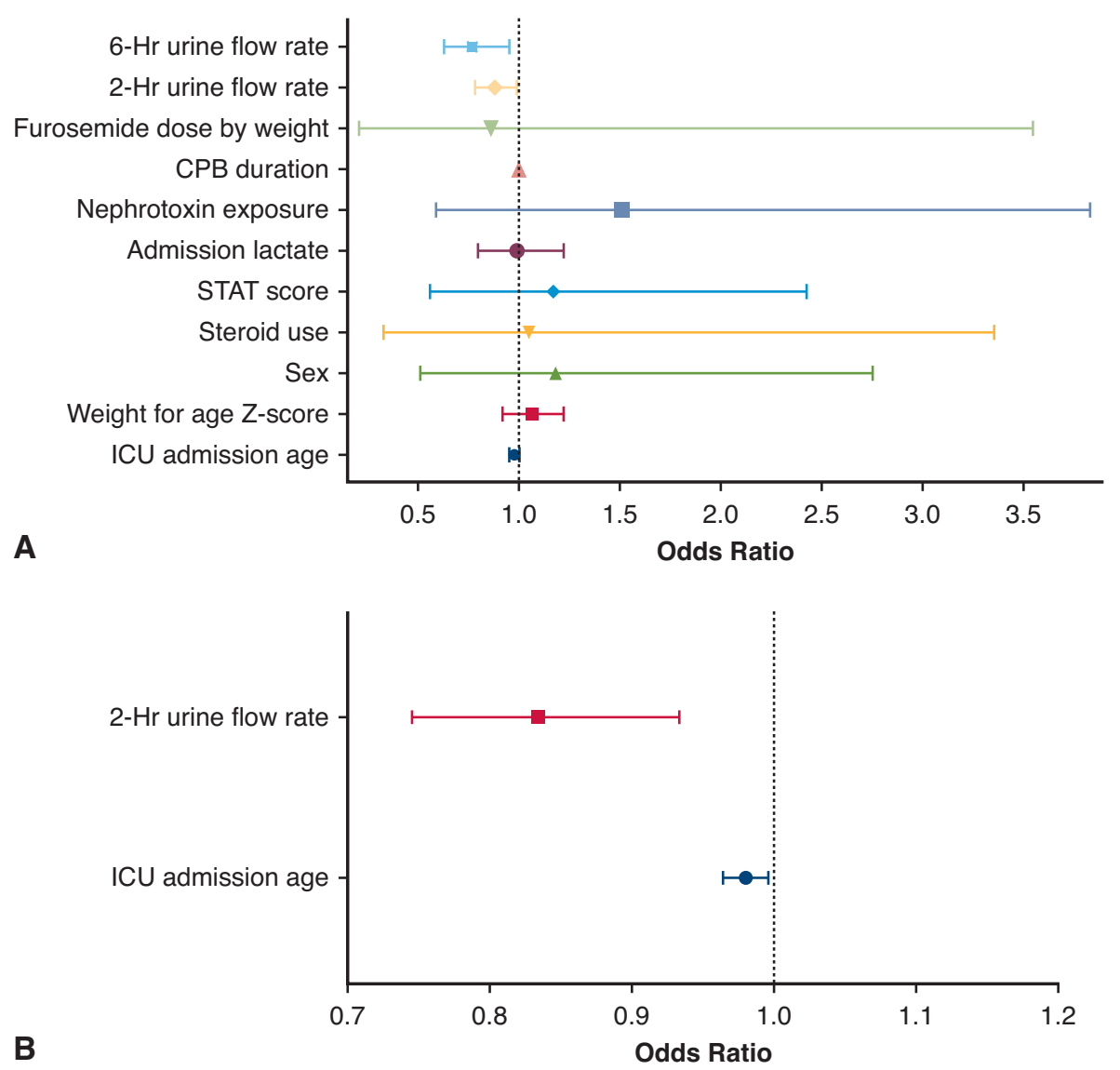

B

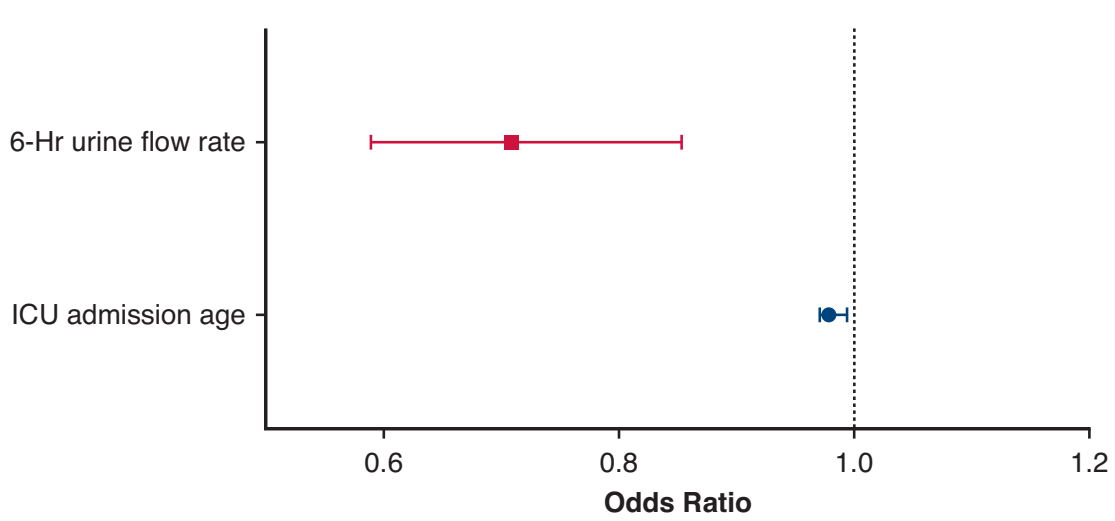

FIGURE 2. Forest plot of the odds ratios and $95 \%$ CIs for the factors associated with AKI. A, Univariate analysis depicting all included variables. B, Multivariable analysis including only variables including 2-hour UFR that are independently associated with AKI. C, Multivariable analysis including only variables with 6-hour UFR that are independently associated with AKI. CPB, Cardiopulmonary bypass; STAT, Society of Thoracic SurgeonsEuropean Association for Cardio-Thoracic Surgery (surgical complexity score); $I C U$, intensive care unit.

predictive values for 2-hour UFR and 6-hour UFR are $92.7 \%$ and $92.9 \%$, respectively. Changes in sensitivity and specificity with varying UFR, along with the optimal cutoff point for predicting severe AKI, are described in Table 3. A second ROC analysis used additional clinical variables, including age, sex, and STAT score. The AUC for 2-hour UFR improved to 0.74 (95\% CI, 0.63-0.85), and the AUC for the 6-hour UFR improved to 0.75 (95\% CI, 0.64-0.83).

\section{DISCUSSION}

This is the first multicenter pediatric study examining the utility of the FST for AKI prediction. In our heterogeneous cohort of children after cardiac surgery, we found the 2- and 
TABLE 3. Sensitivity/specificity for predicting acute kidney injury by urine flow rate

\begin{tabular}{|c|c|c|c|c|}
\hline $\begin{array}{l}\text { Test result } \\
\text { variable(s) }\end{array}$ & $\begin{array}{l}\text { Positive if } \\
\text { less than }\end{array}$ & Sensitivity & Specificity & Youden's J \\
\hline \multirow[t]{12}{*}{ UFR 0-2 h } & 0.5 & 0.15 & 0.94 & 0.095 \\
\hline & 1.0 & 0.27 & 0.87 & 0.137 \\
\hline & 1.5 & 0.39 & 0.81 & 0.194 \\
\hline & 2.0 & 0.50 & 0.79 & 0.287 \\
\hline & 2.5 & 0.54 & 0.74 & 0.273 \\
\hline & 3.0 & 0.65 & 0.68 & 0.338 \\
\hline & 3.5 & 0.69 & 0.64 & 0.332 \\
\hline & 3.6 & 0.73 & 0.64 & 0.371 \\
\hline & 4.0 & 0.73 & 0.60 & 0.327 \\
\hline & 4.5 & 0.73 & 0.53 & 0.260 \\
\hline & 5.0 & 0.77 & 0.48 & 0.247 \\
\hline & 5.5 & 0.81 & 0.44 & 0.249 \\
\hline \multirow[t]{12}{*}{ UFR 0-6 h } & 0.5 & 0.04 & 0.96 & -0.006 \\
\hline & 1.0 & 0.31 & 0.93 & 0.234 \\
\hline & 1.5 & 0.31 & 0.85 & 0.154 \\
\hline & 2.0 & 0.50 & 0.78 & 0.279 \\
\hline & 2.5 & 0.65 & 0.70 & 0.353 \\
\hline & 2.7 & 0.73 & 0.68 & 0.407 \\
\hline & 3.0 & 0.77 & 0.60 & 0.365 \\
\hline & 3.5 & 0.81 & 0.54 & 0.345 \\
\hline & 4.0 & 0.81 & 0.46 & 0.264 \\
\hline & 4.5 & 0.81 & 0.39 & 0.198 \\
\hline & 5.0 & 0.81 & 0.35 & 0.154 \\
\hline & 5.5 & 0.89 & 0.29 & 0.172 \\
\hline
\end{tabular}

UFRs at incrementally increasing rates are listed in the second column in $\mathrm{mL} / \mathrm{kg} / \mathrm{h}$ through 2 and 6 hours. Best sensitivity/specificity for UFR is highlighted. UFR, Urine flow rate.

6-hour UFR after a dose of furosemide was associated with the development of creatinine-defined AKI, even after adjusting for confounding variables. The odds of developing worse AKI increased as the UFR decreased. Lower 2- and 6-hour UFR were associated with a longer length of stay, irrespective of AKI status, duration of mechanical ventilation, surgical complexity, and patient demographics.

Our findings are consistent with 2 retrospective, singlecenter studies of infants after cardiac surgery. As our results showed, those studies demonstrated that lower UFR was associated with subsequent development of AKI. ${ }^{13,14}$ Our study is unique in that this is the first multicenter study showing that these findings are generalizable. We also expanded the age range to which these results may apply because previous articles only included infants, whereas the mean age in our cohort was more than 6 months. Furthermore, when we removed infants from our analysis, the associations persisted, showing that infant age group did not drive the association. Finally, we did not use a predetermined criterion for low UFR after furosemide, rather, we report the observed sensitivity/specificity across a wide range of incremental increases or decreases in UFR. We determined that the UFR changed the odds of AKI, meaning the odds of developing more severe AKI increased as the UFR decreased. Using higher and lower cutoffs would improve sensitivity and specificity, respectively, although the negative predictive value at these cutoffs is already good in this cohort. When we applied a cutoff point less than $1 \mathrm{~mL} / \mathrm{kg} / \mathrm{h}$ for 2- and 6hour UFR as described by Kakajiwala and colleagues, ${ }^{13}$ for instance, the specificity for predicting severe AKI improved, ranging from $86 \%$ to $92 \%$, respectively.

Compared with our study, performing the FST in critically ill adult patients demonstrates a higher AUC, ranging from 0.82 to $0.87 .^{8}$ In the adult study, all patients tested had at least stage $1 \mathrm{AKI}$ at the time of furosemide affecting the pretest probability of the association between 2-hour UFR and AKI progression. It is also possible that the difference is related to factors that are specific to postcardiac surgery physiology, fluid overload that may dilute $\mathrm{SCr}$ thereby affecting AKI recognition, and inclusion of neonates whose measured creatinine reflects maternal levels if surgery was performed during the first week of life. Dilution of $\mathrm{SCr}$ can mask AKI diagnosis and diminish the strength of the association between AKI and outcomes, implying correction of creatinine for fluid balance may be important in neonates and small children. ${ }^{15}$ The degree of ultrafiltration used in the operating room during and after CPB and the amount of fluid resuscitation in the cardiac intensive care unit will affect the typical intravascular volume status at a given institution and are therefore likely to affect the predictive thresholds of the FST. The cross-institution data theoretically overcome the potential confounder of institutional bias or management tendencies. The predictive ability of the FST may be enhanced by standardizing the dose and time of administration, as well as accounting for the factors described earlier when tested in a prospective manner.

It was interesting that STAT score did not significantly affect the risk of AKI, although we believe we may have limited the effect of STAT by excluding patients with STAT less than 3. We also noted no association with CPB time, although this has been noted previously. ${ }^{14}$ Our ability to detect a difference may have been limited because bypass times could show less variation because we only included more surgically complex patients.

The association between lower UFR and longer length of stay even after adjusting for confounding factors demonstrates the utility of UFR after furosemide as a predictor of a poor outcome. Because the FST was originally conceived as a methodology to test tubular responsiveness, identifying perturbations in this homeostatic mechanism may serve as a marker of progression of illness. 

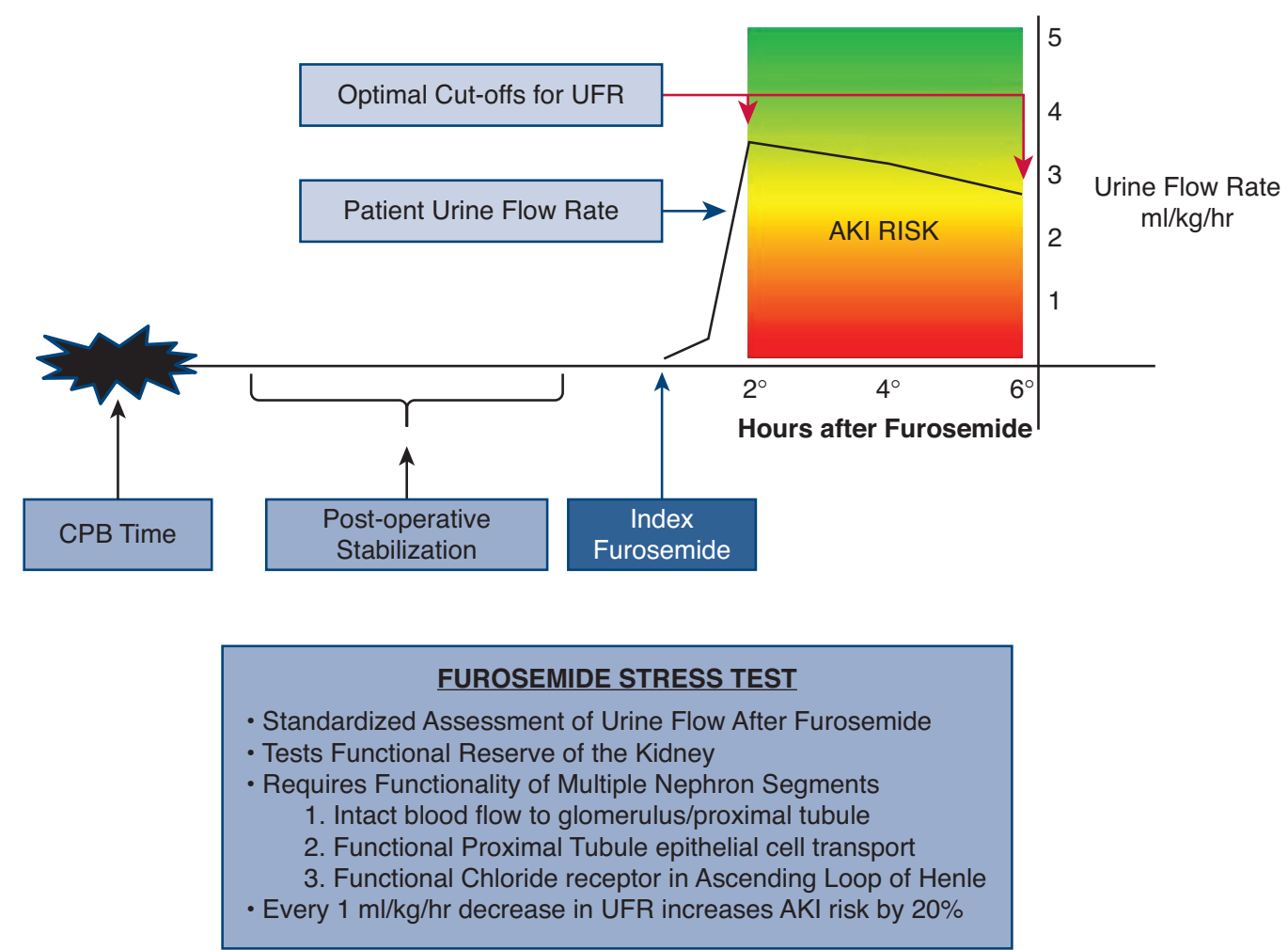

FIGURE 3. Timing of AKI, prognosis using FST, and opportunity to intervene. The window of opportunity to intervene and ameliorate the risk of AKI is shown. On the timeline, CPB is the point at which the injury occurs followed by a period of intensive care unit stabilization. The dose of furosemide is then given that allows for risk stratification by UFR (red indicates higher risk). UFR, Urine flow rate; $A K I$, acute kidney injury; $C P B$, cardiopulmonary bypass.

Several clinically available AKI biomarkers exist and may be helpful in enhancing the predictive performance of the FST. Specifically, urine neutrophil gelatinaseassociated lipocalin, tissue inhibitor metalloproteinase-2, insulin-like growth factor binding protein-7, and cystatin $\mathrm{C}$ have all been shown to predict $\mathrm{AKI}$ in children after cardiac surgery before an increase in SCr. ${ }^{16-19}$ Prospective studies assessing whether AKI biomarkers in conjunction with the FST enhance the prediction of AKI are necessary.

A recent prospective study in pediatric patients after cardiac surgery randomized patients to receive standard diuretic therapy or peritoneal dialysis within 6 hours after cardiac surgery. Kwiatkowski and colleagues ${ }^{20}$ demonstrated a lower rate of fluid overload, a longer duration of ventilation, a longer duration of vasoactive medication use, and increased electrolyte derangements among patients who received furosemide. Although prophylactic peritoneal dialysis may not be suitable for all children undergoing cardiac surgery, risk stratification based on responsiveness to furosemide may identify which patients are most likely to benefit from early peritoneal dialysis. As in our study, prediction of AKI may be further enhanced by considering clinical risk factors such as age, sex, and STAT score. Figure 3 illustrates the idea that if AKI risk can be identified early enough after $\mathrm{CPB}$, we may be able to alter treatment to lessen the risk of development or progression of AKI. This has the potential to reduce duration of ventilation, rates of AKI progression, associated systemic complications, and ultimately hospital costs. ${ }^{21}$ Given the previous studies in infants and this study expanding those results to older age groups across multiple institutions, the next step should be a prospective study to validate the FST. This study will need to include multiple age ranges because furosemide response may vary by age. For instance, UFR found among this group of patients was higher than in 2 previous reports that included only infants. ${ }^{13,14}$

\section{Study Limitations}

This study has several previously mentioned strengths, but there are limitations. The sample size and retrospective nature of the study allow unappreciated variables to affect our results. For instance, additional variables, such as vasoactive inotrope score, temperature nadir during bypass, rewarming temperature, need for blood products, hemoglobin nadir, and preoperative anemia, may be factors that could be included in a prospective study. Given the wide variety of factors that may influence kidney injury, a prospective trial will be better equipped than a retrospective 
trial to try to account for these factors. We could not correct for individual site or practitioner variation in practice, particularly regarding fluid management and AKI prevention or management. As stated before, however, this weakness may increase the applicability of our findings given the ability to overcome single institutional bias with regard to fluid and AKI management. We did not correct creatinine for fluid overload, thereby possibly missing cases of AKI due to dilution of creatinine. Neonates were included, and their SCr before surgery may have still been reflective of maternal levels, thereby masking AKI using currently available definitions. We were not able to assess for degree of capillary leak or presence of low cardiac output at the time of furosemide administration, both of which may affect UFR. Finally, a nonstandardized furosemide dose was administered at varying times after cardiac surgery, which may decrease the discriminatory ability of the test.

\section{CONCLUSIONS}

In this study, lower UFR after furosemide administration in a heterogeneous cohort of children from multiple institutions after CPB was independently associated with subsequent development of AKI and a longer length of hospital stay. Future prospective studies targeted to evaluate the efficacy of the FST for prediction of AKI in children are needed.

\section{Conflict of Interest Statement}

R.K.B. has a consultancy agreement with Bioporto Diagnostics and serves on the Speakers Bureau for Baxter Acute Therapies. These present no relevant conflict of interest for the work presented in this report. All other authors have nothing to disclose with regard to commercial support.

The authors thank Abdul Milad and Julie Connelly, RN, BSN, CCRN, CCRC.

\section{References}

1. Kaddourah A, Basu R, Bagshaw S, Goldstein S. Epidemiology of acute kidney injury in critically ill children and young adults. N Engl J Med. 2017;376:11-20.

2. Jetton J, Boohaker L, Sethi S, Wazir S, Rohatgi S, Soranno D, et al. Incidence and outcomes of neonatal acute kidney injury (AWAKEN): a multicentre, multinational, observational cohort study. Lancet. 2017;1:184-94.

3. Blinder J, Goldstein S, Lee V, Baycroft A, Fraser C, Nelson D, et al. Congenital heart surgery in infants: effects of acute kidney injury on outcomes. $J$ Thorac Cardiovasc Surg. 2012;143:368-74.

4. Li S, Krawczeski C, Zappitelli M, Devarajan P, Thiessen-Philbrook H, Coca S, et al. Incidence, risk factors, and outcomes of acute kidney injury after pediatric cardiac surgery: a prospective multicenter study. Crit Care Med. 2011;39:1493-9.
5. Park S, Hur M, Kim E, Kim W, Park J, Kim Y, et al. Risk factors for acute kidney injury after congenital cardiac surgery in infants and children: a retrospective observational study. PLoS One. 2016;11:e0166328.

6. Alge J, Arthur J. Biomarkers of AKI: a review of mechanistic relevance and potential therapeutic implications. Clin J Am Soc Nephrol. 2015;10:147-55.

7. Meersch M, Schmidt C, Hoffmeier A, Van Aken H, Wempe C, Gerss J, et al Prevention of cardiac surgery-associated AKI by implementing the KDIGO guidelines in high risk patients identified by biomarkers: the PrevAKI randomized controlled trial. Intensive Care Med. 2017;43:1551-61.

8. Koyner J, Davison D, Brasha-Mitchell E, Chalikonda D, Arthur J, Shaw A, et al. Furosemide stress test and biomarkers for the prediction of AKI severity. J Am Soc Nephrol. 2015;26:2023-31.

9. Jacobs J, Jacobs M, Maruszewski B, Lacour-Gayet F, Tchervenkov C, Tobota Z, et al. Initial application in the EACTS and STS congenital heart surgery databases of an empirically derived methodology of complexity adjustment to evaluate surgical case mix and results. Eur $J$ Cardiothorac Surg. 2012;42:775-9.

10. Zappitelli M, Parikh C, Akcan-Arikan A, Washburn K, Moffett B, Goldstein S Ascertainment and epidemiology of acute kidney injury varies with definition interpretation. Clin J Am Soc Nephrol. 2008;3:948-54.

11. Chawla L, Davison D, Brasha-Mitchell E, Koyner J, Arthur J, Shaw A, et al Development and standardization of a furosemide stress test to predict the severity of acute kidney injury. Crit Care. 2013;17:R207.

12. Kidney Disease: Improving Global Outcomes (KDIGO) Acute Kidney Injury Work Group. KDIGO clinical practice guidelines for acute kidney injury. Kidney Int Suppl. 2012;2:1-138.

13. Kakajiwala A, Kim J, Hughes J, Costarino A, Ferguson J, Gaynor J, et al. Lack of furosemide responsiveness predicts acute kidney injury in infants after cardiac surgery. Ann Thorac Surg. 2017;104:1388-94.

14. Borasino S, Wall K, Crawford J, Hock K, Cleveland D, Rahman F, et al Furosemide response predicts acute kidney injury after cardiac surgery in infants and neonates. Pediatr Crit Care Med. 2018;19:310-7.

15. Basu R, Andrew A, Krawczeski C, Manning P, Wheeler D, Goldstein S. Acute kidney injury based on corrected serum creatinine is associated with increased morbidity in children following the arterial switch operation. Pediatr Crit Care Med. 2013;14:e218-24.

16. Krawczeski C, Goldstein S, Woo J, Wang Y, Piyaphanee N, Ma Q, et al Temporal relationship and predictive value of urinary acute kidney injury biomarkers after pediatric cardiopulmonary bypass. J Am Coll Cardiol. 2011;58:2301-9.

17. Zappitelli M, Krawczeski C, Devarajan P, Wang Z, Sint K, ThiessenPhilbrook H, et al. Early postoperative serum cystatin C predicts severe acute kidney injury following pediatric cardiac surgery. Kidney Int. 2011; 80:655-62.

18. Dong L, Ma Q, Bennett M, Devarajan P. Urinary biomarkers of cell cycle arres are delayed predictors of acute kidney injury after pediatric cardiopulmonary bypass. Pediatr Nephrol. 2017:32:2351-60.

19. Gist K, Goldstein S, Wrona J, Alten J, Basu R, Cooper D, et al. Kinetics of the cell cycle arrest biomarkers (TIMP-2*IGFBP-7) for prediction of acute kidney injury in infants after cardiac surgery. Pediatr Nephrol. 2017;32:1611-9.

20. Kwiatkowski D, Goldstein S, Cooper D, Nelson D, Morales D Krawczeski C. Peritoneal dialysis vs furosemide for prevention of fluid overload in infants after cardiac surgery: a randomized clinical trial. JAMA Pediatr. 2017;171:357-64.

21. Kwiatkowski D, Price E, Axelrod D, Romfh A, Han B, Sutherland S, et al Incidence, risk factors, and outcomes of acute kidney injury in adults undergoing surgery for congenital heart disease. Cardiol Young. 2017;27:1068-75.

Key Words: furosemide, acute kidney injury, pediatric cardiac surgery, urine flow rate 